\title{
Observables of Quantum Gravity at the LHC
}

\section{S. Hossenfelder*}

Perimeter Institute for Theoretical Physics

31 Caroline St. N, Waterloo Ontario, N2L 2Y5, Canada

E-mail: sabine@perimeterinstitute.ca

In models with large extra dimensions, the Planck scale can be lowered to values soon accessible at the LHC. These models predict a vast number of quantum gravitational effects at the lowered Planck scale, among them the production of TeV-mass black holes and gravitons. Since the Planck length is expected to set a fundamental limit to the resolution of structures, within this scenario the effects of such a minimal length scale also come into the reach of experiment.

From Quantum to Emergent Gravity: Theory and Phenomenology

June 11-15 2007

Trieste, Italy

${ }^{*}$ Speaker. 


\section{Large Extra Dimensions}

Located between particle physics and general relativity, interactions at the Planck scale represent a future challenge and a key to our understanding of the fundamental laws of nature. Unfortunately, the gravitational interaction is far weaker than the other interactions of the Standard Model (SM). Though it grows more important with increasing energy, extrapolating the gravitational strength to highest energies shows that quantum gravitational effects will become nonnegligible only at energies far outside the reach of future collider experiments, at the so-called Planck scale $m_{\mathrm{p}} \sim 10^{16} \mathrm{TeV}$.

However, this extrapolation over 16 orders of magnitude implicitly assumes that in this range no unexpected modifications occur. Since it is an open question why gravity is so much weaker than the other interactions, one can speculate that it might instead be our extrapolation to higher energies is inappropriate, and quantum gravitational effects will become important much earlier, somewhere around the electroweak scale. A concrete scenario to realize this are models with extra dimensions.

During the last decade, several such models using compactified Large Extra Dimensions (LXDs) as an additional assumption to the quantum field theories of the SM have been proposed. The setup of these effective models is motivated by String Theory though the question whether our spacetime has additional dimensions is well-founded on its own and worth the effort of examination.

The models with LXDs provide us with an useful description to predict first effects beyond the SM. They do by no means claim to be a theory of first principles or a candidate for a grand unification. Instead, their simplified framework allows the derivation of testable results which can in turn help us to gain insights about the underlying theory.

There are different ways to build a model of an extra dimensional space-time. To mention only the most common ones:

1. The ADD-model proposed by Arkani-Hamed, Dimopoulos and Dvali [1] adds $d$ extra spacelike dimensions without curvature, in general each of them compactified to the same radius $R$. All SM particles are confined to our brane, while gravitons are allowed to propagate freely in the bulk.

2. The setting of the model from Randall and Sundrum [2, 3] is a 5-dimensional spacetime with an non-factorizable geometry. The solution for the metric is found by analyzing the solution of EinsteinŠs field equations with an energy density on our brane, where the SM particles live. In the type I model [2] the extra dimension is compactified, in the type II model [3] it is infinite.

3. Within the model of universal extra dimensions [4] all particles (or in some extensions, only bosons) can propagate in the whole multi-dimensional spacetime. The extra dimensions are compactified on an orbifold to reproduce SM gauge degrees of freedom.

In the following we will focus on the model (1) which yields a beautiful and simple explanation of the hierarchy problem. Consider a particle of mass $m$ located in a spacetime of $d+3$ dimensions. 
The general solution of Poisson's equation yields its potential as a function of the radial distance $r$ to the source

$$
V(r) \propto \frac{1}{M_{\mathrm{f}}^{d+2}} \frac{m}{r^{d+1}}
$$

where we have introduced a new fundamental mass-scale $M_{\mathrm{f}}$. The hierarchy problem then is the question why, for $d=0$, this mass-scale is the Planck mass, $m_{\mathrm{p}}$, and by a factor $10^{16}$ smaller than the mass-scales in the SM, e.g. the weak scale.

The additional $d$ spacetime dimensions are compactified on radii $R$, which are small enough to have been unobserved so far. Then, at distances $r \gg R$, the extra dimensions will 'freeze out' and the potential Eq. (1.1) will turn into the common $1 / r$ potential, but with a prefactor given by the volume of the extra dimensions

$$
V(r) \rightarrow \frac{1}{M_{\mathrm{f}}^{d+2}} \frac{1}{R^{d}} \frac{m}{r} .
$$

In this limit, we will rediscover the usual gravitational law which yields the relation

$$
m_{\mathrm{p}}^{2}=M_{\mathrm{f}}^{d+2} R^{d}
$$

Given that $M_{\mathrm{f}}$ has the right order of magnitude to be compatible with the other observed scales, it can be seen from this argument that the volume of the extra dimensions suppresses the fundamental scale and thus, explains the huge value of the Planck mass.

The radius $R$ of these extra dimensions, for $M_{\mathrm{f}} \sim \mathrm{TeV}$, can be estimated with Eq.(1.3) and typically lies in the range from mm to $10^{3} \mathrm{fm}$ for $d$ from 2 to 7 , or the inverse radius $1 / R$ lies in energy range $\mathrm{eV}$ to $\mathrm{MeV}$, respectively. The case $d=1$ is excluded. It would result in an extra dimension about the size of the solar system.

Due to the compactification, momenta in the direction of the LXDs can only occur in quantized steps $\propto 1 / R$ for every particle which is allowed to enter the bulk. The fields can be expanded in Fourier-series

$$
\psi(x, y)=\sum_{n=-\infty}^{+\infty} \psi^{(n)}(x) \exp (\mathrm{i} n y / R)
$$

where $x$ are the coordinates on our brane and $y$ the coordinates of the LXDs. This yields an infinite number of equally spaced excitations, the so called Kaluza-Klein-Tower. On our brane, these massless KK-excitations act like massive particles, since the momentum in the extra dimensions generates an apparent mass term

$$
\left[\partial_{x} \partial^{x}-\left(\frac{n}{R}\right)^{2}\right] \psi^{(n)}(x)=0
$$

\section{Phenomenology}

The most obvious experimental test for the existence of extra dimensions is a measurement of the Newtonian potential at sub-mm distances. Cavendish-like experiments which search for 
deviations from the $1 / r$ potential have been performed during the last years with high precision [5]. No deviations have been found, which constrains the case with $\mathrm{d}=2$.

Also the consequences for high energy experiments are intriguing. Since the masses of the KK-modes are so low, they get excited easily but it is not until energies of order $M_{\mathrm{f}}$ that their phase-space makes them give an important contribution in scattering processes. The number of excitations $N(\sqrt{s})$ below an energy $\sqrt{s}^{1}$ can, for an almost continuous spectrum, be estimated with the volume of the $d$-dimensional sphere of radius $R \sqrt{s}$. We can then estimate the total crosssection for a point interaction, e.g. $e^{+} e^{-} \rightarrow G \gamma$ ( $G$ denotes the graviton) by

$$
\sigma\left(e^{+} e^{-} \rightarrow G \gamma\right) \approx \frac{\alpha}{m_{\mathrm{p}}^{2}} N(\sqrt{s})=\frac{\alpha}{s}\left(\frac{\sqrt{s}}{M_{\mathrm{f}}}\right)^{d+2},
$$

where we have used Eq.(1.3). As can be seen, at energy scales close to the new fundamental scale the estimated cross-section becomes comparable to cross-sections of electroweak processes.

The necessary Feynman rules for exact calculations of the graviton tree-level interactions have be derived [6] and the cross-sections have been examined closely. Since the gravitons are not detected, their emission would lead to an energy loss in the collision and to a higher number of monojets. Modifications of SM predictions do also arise by virtual graviton exchange, which gives additional contributions in the calculation of cross-sections.

Another exciting signature of LXDs is the possibility of black hole production. In the standard $3+1$ dimensional space-time, the production of black holes requires a concentration of energydensity which can not be reached in the laboratory. As we have seen, in the higher dimensional space-time, gravity becomes stronger at small distances and therefore the event horizon is located at a larger radius. We can estimate the horizon radius, $R_{H}$, of a mass $m$ by using the Newtonian potential Eq. (1.1), where we will assume that the black hole is small enough to completely fit into the extra dimensions $R_{H} \ll R$. In the Newtonian limit, the radial entry of the metric tensor is approximately given by $g_{r r}=1-2 V(r)$, and the horizon appears at the zero of $g_{r r}$ which leads to

$$
R_{H} \sim \frac{1}{M_{\mathrm{f}}}\left(\frac{m}{M_{\mathrm{f}}}\right)^{\frac{1}{d+1}} .
$$

The exact formula which can be derived from the higher dimensional Schwarzschild-metric [7] differs from the given one by some numerical coefficients. It is not surprising to see that a black hole with a mass about the new fundamental mass $m \approx M_{\mathrm{f}}$, has a radius of about the new fundamental length scale $L_{\mathrm{f}}=1 / M_{\mathrm{f}}$ (which justifies the use of the limit $R_{H} \ll R$ ). For $M_{\mathrm{f}} \sim 1 \mathrm{TeV}$ this radius is $\sim 10^{-4} \mathrm{fm}$. Thus, at the LHC it would be possible to bring particles closer together than their Schwarzschild-radius, and a black hole could be created. Black holes with masses in the range of the lowered Planck scale should be a subject of quantum gravity. Since there is yet no theory available to perform this analysis, the black holes are treated as semi classical objects which form intermediate metastable states.

To compute the production details, the cross-section of the black holes can be approximated by the classical geometric cross-section

$$
\sigma(M) \approx \pi R_{H}^{2}
$$

\footnotetext{
${ }^{1}$ The estimated center of mass energy for the LHC is $\sqrt{s} \approx 14 \mathrm{TeV}$.
} 
an expression which contains only the fundamental Planck scale as coupling constant. This cross section has been under debate [8], but further investigations justify the use of the classical limit at least up to energies of $\approx 10 M_{\mathrm{f}}$ [9]. It has further been shown that the naively expected classical result remains valid also in String Theory [17]. However, the topic is still under discussion, see also the very recent contributions [10].

A common approach to improve the naive picture of colliding point particles, is to treat the creation of the horizon as a collision of two shock fronts in an Aichelburg-Sexl geometry describing the fast moving particles [15]. Due to the high velocity of the moving particles, space time before and after the shocks is almost flat and the geometry can be examined for the occurrence of trapped surfaces.

These semi classical considerations do also give rise to form factors which take into account that not the whole initial energy is captured behind the horizon. These factors have been calculated in [16], depend on the number of extra dimensions, and are of order one. Setting $M_{\mathrm{f}} \sim 1 \mathrm{TeV}$ and $d=2$ one finds $\sigma \approx 1 \mathrm{TeV}^{-2} \approx 400 \mathrm{pb}$. With this it is further found that these black holes will be produced at LHC in number of $\approx 10^{9}$ per year [11]. Once produced, the black holes will undergo an evaporation process whose thermal properties carry information about $M_{\mathrm{f}}$ and $d$. Furthermore, crossing the threshold for black hole production causes a sharp cut-off for high energetic jets as those jets now end up as black holes instead, and are re-distributed into thermal particles of lower energies. Thus, black holes will give a clear signal. For reviews on TeV-scale black holes see [12].

When working on the phenomenology at the Planck scale, one furthermore has to take into account that the Planck length is expected to act as a fundamentally finite resolution of structures; it represents a minimal length scale. A lowering of the fundamental scale then implies a raising of the minimal length. Thus, within the model of LXDs not only the above discussed production of gravitons and black holes occurs at the lowered Planck scale $M_{\mathrm{f}}$, but so do the effects of the minimal length scale.

To incorporate the notion of a minimal length into ordinary quantum field theory one can use a simple model which has been worked out in detail in $[18,19]$. This presence of a minimal length results in a generalized form of the uncertainty principle, and a modified measure on momentum space, which influences cross-sections at Planckian energies. This model is closely related [20] to Deformations of Special Relativity, though differences between both exist. Most notably, the minimal length model does not imply an energy dependent speed of light, and the propagator is modified only off-shell.

As we have seen, the LXD-model predicts a rich phenomenology. Presently available data from collider physics as well as from astrophysics set constraints on the parameters of the model [13]. The most recent constraints can be found in the Particle Data Booklet [14].

\section{Acknowledgements}

I want to thank the organizers of the Trieste Workshop From Quantum to Emergent Gravity for the hospitality. Research at Perimeter Institute for Theoretical Physics is supported in part by the Government of Canada through NSERC and by the Province of Ontario through MRI. 


\section{References}

[1] N. Arkani-Hamed, S. Dimopoulos and G. R. Dvali, Phys. Lett. B 429, 263 (1998); I. Antoniadis, N. Arkani-Hamed, S. Dimopoulos and G. R. Dvali, Phys. Lett. B 436, 257 (1998); N. Arkani-Hamed, S. Dimopoulos and G. R. Dvali, Phys. Rev. D 59, 086004 (1999).

[2] L. Randall and R. Sundrum, Phys. Rev. Lett. 83, 4690 (1999).

[3] L. Randall and R. Sundrum, Phys. Rev. Lett. 83, 3370 (1999).

[4] T. Appelquist, H. C. Cheng and B. A. Dobrescu, Phys. Rev. D 64, 035002 (2001); C. Macesanu, C. D. McMullen and S. Nandi, Phys. Rev. D 66, 015009 (2002); T. G. Rizzo, Phys. Rev. D 64, 095010 (2001).

[5] J. C. Long and J. C. Price, Comptes Rendus Physique 4, 337 (2003); C. D. Hoyle et al, Phys. Rev. D 70, 042004 (2004); C. D. Hoyle et al, Phys. Rev. Lett. 86, 1418 (2001); J. Chiaverini et al, Phys. Rev. Lett. 90, 151101 (2003).

[6] J. Hewett and M. Spiropulu, Ann. Rev. Nucl. Part. Sci. 52, 397 (2002); T. G. Rizzo, Phys. Rev. D 64, 095010 (2001); T. Han, J. D. Lykken and R. J. Zhang, Phys. Rev. D 59 (1999) 105006; S. Cullen, M. Perelstein and M. E. Peskin, Phys. Rev. D 62, 055012 (2000).

[7] R. C. Myers and M. J. Perry Ann. Phys. 172, 304-347 (1986).

[8] M. B. Voloshin, Phys. Lett. B 518, 137 (2001); Phys. Lett. B 524, 376 (2002); S. B. Giddings, in Proc. of the APS/DPF/DPB Summer Study on the Future of Particle Physics (Snowmass 2001) ed. N. Graf, eConf C010630, P328 (2001).

[9] S. N. Solodukhin, Phys. Lett. B 533, 153 (2002); A. Jevicki and J. Thaler, Phys. Rev. D 66, 024041 (2002); T. G. Rizzo, in Proc. of the APS/DPF/DPB Summer Study on the Future of Particle Physics (Snowmass 2001) ed. N. Graf, eConf C010630, P339 (2001); D. M. Eardley and S. B. Giddings, Phys. Rev. D 66, 044011 (2002).

[10] V. S. Rychkov, Phys. Rev. D 70, 044003 (2004); K. Kang and H. Nastase, Phys. Rev. D 71, 124035 (2005).

[11] S. Dimopoulos and G. Landsberg Phys. Rev. Lett. 87, 161602 (2001); P.C. Argyres, S. Dimopoulos, and J. March-Russell, Phys. Lett. B441, 96 (1998).

[12] P. Kanti, Int. J. Mod. Phys. A 19, 4899 (2004); G. Landsberg, [hep-ph/0211043]; M. Cavaglia, Int. J. Mod. Phys. A 18, 1843 (2003); S. Hossenfelder, in 'Focus on Black Hole Research', pp. 155-192, Nova Science Publishers (2005).

[13] K. Cheung, [arXiv:hep-ph/0409028].

[14] W.-M. Yao et al., J. Phys. G 33, 1 (2006), website: pdg . lbl •gov

[15] I. Ya. Yref'eva, Part.Nucl. 31, 169-180 (2000). S. B. Giddings and V. S. Rychkov, Phys. Rev. D 70, 104026 (2004); V. S. Rychkov, [arXiv:hep-th/0410041]; T. Banks and W. Fischler, [arXiv:hep-th/9906038]. O. V. Kancheli, [arXiv:hep-ph/0208021].

[16] H. Yoshino and Y. Nambu, Phys. Rev. D 67, 024009 (2003).

[17] G. T. Horowitz and J. Polchinski, Phys. Rev. D 66103512 (2002).

[18] S. Hossenfelder et al, Phys. Lett. B 575, 85 (2003).

[19] S. Hossenfelder, Phys. Rev. D 70, 105003 (2004) [arXiv:hep-ph/0405127].

[20] S. Hossenfelder, Class. Quant. Grav. 23, 1815 (2006) [arXiv:hep-th/0510245].

[21] S. Hossenfelder, Phys. Rev. D 73, 105013 (2006) [arXiv:hep-th/0603032]. 